\title{
Fatigue life of oil palm wood (OPW) for furniture applications
}

\begin{abstract}
Oil palm wood (OPW) from different portions of the trunk was tested on edge to evaluate its fatigue life, a criterion necessary for furniture application. It was found that as the stress levels increased, as a percentage of its MOR, a reduction in fatigue life was observed. Further, the phenol-formaldehyde resin impregnated OPW from the middle and center portion of the oil palm trunk showed comparable fatigue life with those untreated samples from the periphery of the trunk, and hence, the allowable design stress for the OPW for furniture applications could be set at $40 \%$ of its MOR. The results show oil palm wood of acceptable density can be successfully used for furniture applications.
\end{abstract}

Keyword: Fatigue life; Vascular bundle; Formaldehyde resin; Thick cell wall; High strength property 